\title{
EFEITO DO CULTIVO, ADUBAÇĀO E CARACTERES VARIETAIS NA PRODUTIVIDADE DO AMENDOINZEIRO (Arachis hypogaea L.) *
}

\author{
Paulo R.C. Castro ** \\ Leonardo Zanata****
}

RESUMO

Verificaram-se os efeitos do cultivo, adubação e caracteres varietais na produtividade biológica do amendoinzeiro em condições de campo, na região de Jaboticabal (SP).

Testou-se a efetividade da prática da amontoa, da adubação 9:30:16 com N-P-K (250 kg/ha) e da cultivar. "Tatu-53". em relação à linhagem local melhorada ("Tatu - Lusitânia") .

As produções em quilograma por hectare de vagens ou de grãos de amendoim não foram significativamente afetadas pelas práticas em estudo. Observou-se porém aumento na produtividade de vagens e de grãos na cultivar "Tatu - Lusitânia" quando comparada com "Tatu - 53").

Notaram-se que as práticas de adubação e cultivo, isolada ou conjuntamente, proporcionaram uma tendência de aumènto no número de vagens de amendoim por hectare; sendo que a produção de hastes e folhas revelou-se mais elevada na cultivar “Tatu - Lusitânia”.

\section{INTRODUÇÃO}

Ensaios efetuados com 16 diferentes cultivares de amendoinzeiro demonstraram que o 'Roxo-40', 'Roxo-54' e 'Tatuí-76' apresentaram maior produtividade, superior mesmo que o 'Tatu-53', nas condições do Estado de São Paulo (CANECCHIO F. ${ }^{\circ}$ et alii, 1967) . A cultivar 'Tatu-53' continua porém tendo boa receptividade entre os agricultores das principais zonas de produção, devido principalmente a fatores ligados à comercialização do produto.

* Entregue para publicação em 7-12-1977

** Departamento de Botânica. E.S.A. "Luiz de Queiroz" - USP.

*** Engenheiro Agrônomo. 
Ao mesmo tempo que se executam as capinas na cultura do amendoinzeiro, deve-se procurar fazer uma amontoa, sem necessidade porém de executá-la como um trato cultural distinto e especializado. Em caso de solo muito argiloso, pode-se fazer uma amontoa especial na ocasião em que as flores, na base da planta, começam a murchar. (GRANER \& GODOY JR., 1967).

GORE (1941), verificou, em ensaios realizados com a cultivar 'Spanish', que a melhor reação à fertilizantes deu-se quando se aplicou $450 \mathrm{~kg} /$ ha de uma formulação completa 6:6:6 para $\mathrm{N}, \mathrm{P}_{2} \mathrm{O}_{5}$ e $\mathrm{K}_{2} \mathrm{O}$. Já para as cultivares tipo Runner, não encontrou reação para $0 \mathrm{~N}$, caso em que se considerou mais adequada a aplicação de $170 \mathrm{~kg}$ de superfosfato e $56 \mathrm{~kg}$ de cloreto de potássio por hectare.

GREENWOOD (1951) observou efeitos significativos na produção de vagens com aplicação de fósforo; o nitrogênio mostrou efeitos variáveis; sendo que não verificou resposta para o potássio.

BEATTIE et alii (1953) consideram que embora o amendoinzeiro seja capaz de fixar nitrogênio atmosférico, trata-se de uma cultura capaz de reduzir consideravelmente o nível de nutrientes do solo. Assim, uma cultura produzindo cerca de $1000 \mathrm{~kg}$ de amendoim descascado e $2000 \mathrm{~kg}$ de feno, retira $65 \mathrm{~kg}$ de $\mathrm{N}, 11 \mathrm{~kg}$ de $\mathrm{P}, 50 \mathrm{~kg}$ de K e 40 $\mathrm{kg}$ de $\mathrm{Ca}$ do solo.

Observou-se que a cultura do amendoinzeiro pode retirar $300 \mathrm{~kg}$ de nitrogênio, $30 \mathrm{~kg}$ de fósforo e $150 \mathrm{~kg}$ de potássio por hectare. Em Israel, a aplicação de $140 \mathrm{~kg}$ / ha de $\mathrm{N}$ elevou de 1,1 para 4 toneladas a produção de amendoim em solos arenosos. O fósforo é particularmente importante no período de formação dos frutos a o potássio na fase vegetativa do ciclo da planta. No Suriname aplicam-se $180 \mathrm{~kg} / \mathrm{ha}$ da fórmula 5:10:10; sendo que em Israel utilizam-se 90 a $180 \mathrm{~kg}$ de $\mathrm{N} / \mathrm{ha}, 155$ a $140 \mathrm{~kg}$ de $\mathrm{P} /$ ha e $40 \mathrm{~kg}$ de $\mathrm{K} / \mathrm{ha}$, segundo o tipo de solo (MALAVOLTA, 1963).

GARGANTINI et alii (1958) verificaram que o elemento responsável pela maior produção do amendoinzeiro 'Tatuí-76' foi o fósforo, vindo a seguir o nitrogênio, sendo que o potássio não afetou a produtividade. Concluíram que a aplicação de 30 e 240 quilos de $\mathrm{N}$ e $\mathrm{P}_{2} \mathrm{O}_{5}$ por hectare é a mais efetiva fórmula a ser empregada.

Experimentos conduzidos em solos podzolizados para estudar o efeito da adubação N-P-K no amendoinzeiro, mostraram que o $\mathbf{P}$ aumentou apreciavelmente a produção em três ensaios, o $\mathrm{N}$ em um e o $\mathrm{K}$ em nenhum deles (TELLA et alii, 1970) .

Ensaios realizados em Latossolo Roxo, demonstraram que $\mathrm{N}$ proporcionou aumentos significativos na produção de amendoinzeiro em três experimentos, o $\mathrm{K}$ em dois e o $\mathrm{P}$ em apenas um deles (TELLA et alii, 1971). 
NAKAGAWA et alii (1973) notaram efeitos do nitrogênio, do fósforo e da interação $\mathrm{N} \times \mathrm{P}$ na produção de vagens do amendoinzeiro; sendo que o potássio não ocasionou alterações significativas na produção da cultivar 'Tatu-53' nestas condições de solo podzolizado.

O objetivo deste ensaio foi verificar o efeito do cultivo e adubação na produtividade de duas linhagens de amendoinzeiro (Anachis hypogaea).

\section{MATERIAL E MÉTODOS}

O ensaio foi realizado em um Latossolo Roxo na região de Jaboticabal, sendo que a semeadura foi efetuada em $6 / 3 / 1972$, obedecendo o espaçamento de $0,60 \mathrm{~m}$ entre linhas e $0,10 \mathrm{~m}$ entre plantas.

Utilizaram-se sementes tratadas com fungicida, tendo-se efetuado o controle preventivo de pragas e doenças, além de se realizar os demais tratos culturais, no decorrer do ciclo do amendoinzeiro.

Foi utilizado um delineamento fatorial com 3 blocos constituídos de 8 parcelas cada um. Estas parcelas, distribuídas ao acaso dentro de cada bloco, foram as seguintes:

1) $\mathrm{C}_{1} \quad \mathbf{F}_{0} \quad \mathrm{~A}_{0}$

2) $C_{1} \quad F_{0} \quad A_{1}$

3) $\mathrm{C}_{1} \mathrm{~F}_{1} \mathrm{~A}_{0}$

4) $\mathrm{C}_{1} \mathrm{~F}_{1} \mathrm{~A}_{1}$

5) $C_{2} \quad F_{0}, A_{0}$

6) $C_{2} \quad F_{0} \quad A_{1}$

7) $C_{2}, F_{1} A_{0}$

8) $C_{2} \quad F_{1} A_{1}$

Onde,

$\mathrm{C}_{1}=$ cultivar 'Tatu-53'

$C_{.2}=$ cultivar 'Tatu-Lusitânia'

$\mathbf{F}_{0}=$ sem aplicação de fertilizante

$F_{1}=$ com aplicação de fertilizante

$A_{11}=$ sem realização de amontoa

$\mathrm{A}_{1}=$ com realização de amontoa

A área total do ensaio abrangeu $502 \mathrm{~m}^{2}$, porém, devido às bordaduras, ficou reduzida a uma área útil de $216 \mathrm{~m}^{2}$; sendo que a área útil de cada bloco foi de $72 \mathrm{~m}^{2}$ e de cada parcela de $9 \mathrm{~m}^{2}$.

Efetuou-se a análise estatística referente ao peso das vagens e ao peso de grãos produzidos em $\mathrm{kg}$ / ha nas 8 parcelas constituintes dos 3 blocos. 
O número de vagens produzidas por hectare nas 8 parcelas foi colocado em histograma, sendo que a produção de hastes e folhas (palhada) em kg por hectare, nas duas cultivares, com e sem fertilizante, foi apresentada também na forma de histograma.

$\mathrm{Na}$ forma gráfica apresentou-se o peso da matéria seca, em g, das. hastes e folhas, separadamente, para as duas cultivares, com e sem fertilizante, no decorrer do ciclo do amendoinzeiro; sendo as determinações do peso da matéria seca, em estufa a $80^{\circ} \mathrm{C}$, realizadas com intervalos de 14 dias.

Para a execução do presente trabalho utilizaram-se sementes do amendoinzeiro 'Tatu-53' e sementes oriundas de culturas de 'Tatu-53' submetidas a seleção massal por agricultores do Bairro de Lusitânia no Município de Jaboticabal (SP) .

As plantas originárias da seleção apresentaram porte ereto, semelhante ao 'Tatu-53', mostraram porém maior desenvolvimento vegetativo, hastes, folhas e frutos com maiores dimensões, apresentaram ainda grãos mais desenvolvidos. Para facilidade de apresentação vamos. nos referir a esta cultivar como 'Tatu-Lusitânia' até que a mesma seja adequadamente descrita, pois ela não apresenta as mesmas características das cultivares já estudadas por CONAGIN (1958) e por outros. autores.

A adubação foi realizada nos sulcos de plantio, numa razão de 250 $\mathrm{kg} / \mathrm{ha}$ da fórmula 9:30:16 de N-P-K.

A amontoa foi executada manualmente, com auxílio de enxadas, 15 dias após o aparecimento das primeiras flores.

\section{RESULTADOS E DISCUSSÃO}

TABELA 1 - Peso das vagens de amendoim produzidas em $\mathrm{kg} / \mathrm{ha}$ nos 8 tratam atos, em 3 blocos; médias de produção e análise de variância.

\begin{tabular}{ccccc}
\hline Tratamentos & Bloco 1 & Bloco 2 & Bloco 3 & Médias \\
\hline $\mathrm{C}_{1} \mathrm{~F}_{0} \mathrm{~A}_{0}$ & 694 & 1333 & 1847 & 1291 \\
$\mathrm{C}_{1} \mathrm{C}_{0} \mathrm{~A}_{1}$ & 1472 & 1375 & 1763 & 1537 \\
$\mathrm{C}_{1} \mathrm{~F}_{1} \mathrm{~A}_{0}$ & 1763 & 1541 & 1416 & 1573 \\
$\mathrm{C}_{1} \mathrm{~F}_{1} \mathrm{~A}_{1}$ & 1958 & 1791 & 2069 & 1939 \\
$\mathrm{C}_{2} \mathrm{~F}_{0} \mathrm{~A}_{0}$ & 2083 & 2985 & 3221 & 2763 \\
$\mathrm{C}_{2} \mathrm{~F}_{0} \mathrm{~A}_{1}$ & 3916 & 4290 & 2499 & 3568 \\
$\mathrm{C}_{2} \mathrm{~F}_{1} \mathrm{~A}_{0}$ & 3652 & 2291 & 2916 & 2953 \\
$\mathrm{C}_{2} \mathrm{~F}_{1} \mathrm{~A}_{1}$ & 4304 & 2097 & 3388 & 3263 \\
\hline
\end{tabular}




\begin{tabular}{lcc}
\hline Causa de Variação & GL & F \\
\hline Tratamentos & 7 & $5,03^{* *}$ \\
Blocos & 2 & 0,32 \\
Resíduo & 14 & - \\
\hline Total & 23 & - \\
\hline
\end{tabular}

(**) Significativo ao nível de $1 \%$.

Como os tratamentos diferem entre si ao nível de $1 \%$ de probabilidade, efetuou-se um desdobramento dos graus de liberdade, estudando-se os efeitos do fertilizante, cultivar e amontoa, isolada e conjuntamente. Os resultados encontram-se na tabela 2.

TABELA 2 - Análise de variância do peso das vagens de amendoim produzidas em $\mathrm{kg} / \mathrm{ha}$, com desdobramento dos graus de liberdade.

\begin{tabular}{lcc}
\hline Causa de Variação & GL & F \\
\hline Fertilizante (F) & 1 & 0,2639 \\
Cultivar (C) & 1 & $31,37^{* *}$ \\
Amontoa (A) & 1 & 2,43 \\
F x C & 1 & 0,5211 \\
F x A & 1 & 0,1143 \\
C x A & 1 & 0,2068 \\
F x C x A & 1 & 0,3089 \\
\hline (Tratamentos) & $(7)$ & - \\
Blocos & 2 & 0,3213 \\
Resíduo & 14 & - \\
\hline Total & 23 & - \\
\hline
\end{tabular}

(**) Significativo ao nível de $1 \%$.

O coeficiente de variação foi da ordem de $28,74 \%$.

De acordo com a tabela 1 verificamos que os tratamentos diferem entre si ao nível de $1 \%$ de probabilidade. Quando se efetuou o desdobramento dos graus de liberdade observamos diferença significativa apenas entre a produtividade de vagens das duas cultivares em estudo; sendo que a 'Tatu-Lusitânia' mostrou-se mais produtiva que a 'Tatu-53' (tabela 2).

Não se verificaram diferenças significativas na produção de vagens com a utilização de fertilizantes ou com a prática da amontoa, sendo que essas diferenças não ocorreram mesmo quando se considerou a interação entre os tratamentos aplicados (tabela 2). 
TABELA 3 - Peso dos grãos de amendoim produzidos em $\mathrm{kg} / \mathrm{ha}$ nos 8 tratamentos, em 3 blocos; médias de produção e análise de variância.

\begin{tabular}{|c|c|c|c|c|}
\hline Tratamentos & Bloco 1 & Bloco 2 & Bloco 3 & Médias \\
\hline $\mathrm{C}_{1} \mathrm{~F}_{0} \mathrm{~A}_{0}$ & 472 & 903 & 1139 & 838 \\
\hline $\mathrm{C}_{1} \mathrm{C}_{0} \mathrm{~A}_{1}$ & 1014 & 944 & 1194 & 1051 \\
\hline$C_{1} F_{1} A_{0}$ & 1222 & 1041 & 958 & 1074 \\
\hline$C_{1} F_{1} A_{1}$ & 1069 & 1222 & 1430 & 1240 \\
\hline $\mathrm{C}_{2} \mathrm{~F}_{0} \mathrm{~A}_{0}$ & 1389 & 1972 & 2208 & 1856 \\
\hline $\mathrm{C}_{2} \mathrm{~F}_{0} \mathrm{~A}_{1}$ & 2666 & 2916 & 1888 & 2490) \\
\hline$C_{2} F_{1} A_{0}$ & 2430 & 1486 & 1902 & 1939 \\
\hline $\mathrm{C}_{2} \mathrm{~F}_{1} \mathrm{~A}_{1}$ & 2930 & 1444 & 2222 & 2199 \\
\hline
\end{tabular}

\begin{tabular}{lcc}
\hline Causa de Variação & GL & F \\
\hline Tratamentos & 7 & $5,74^{* *}$ \\
Blocos & 2 & 0,28 \\
Resíduo & 14 & - \\
\hline Total & 23 & - \\
\hline
\end{tabular}

(**) Significativo ao nível de $1 \%$.

Devido os tratamentos diferirem entre si ao nível de $1 \%$ de probabilidade, efetuou-se um desdobramento dos graus de liberdade, determinando-se os efeitos do fertilizante, cultivar e amontoa, isolada e conjuntamente. Os resultados encontram-se na tabela 4.

TABELA 4 - Análise de variância do peso dos grãos de amendoim produzidos em $\mathrm{kg} / \mathrm{ha}$, com desdobramento dos graus de liberdade.

\begin{tabular}{lcc}
\hline Causa de Variação & GL & F \\
\hline Fertilizante (F) & 1 & 0,0903 \\
Cultivar (C) & 1 & $35,18^{* *}$ \\
Amontoa (A) & 1 & 3,107 \\
F x C & 1 & 0,7707 \\
F x A & 1 & 0,3391 \\
C x A & 1 & 0,5064 \\
F x C x A & 1 & 0,2069 \\
\hline (Tratamentos) & $(7)$ & - \\
Blocos & 2 & 0,2865 \\
Resíduo & 14 & - \\
\hline Total & 23 & - \\
\hline
\end{tabular}

(**) Significativo ao nível de $1 \%$. 
O coeficiente de variação foi de $27,87 \%$.

Pela tabela 3 observamos que os tratamentos diferem ao nível de $1 \%$ de probabilidade. Com o desdobramento dos graus de liberdade verificamos diferença significativa somente entre a produtividade de grãos das duas cultivares em estudo; sendo que a 'Tatu-Lusitânia' revelou-se mais produtiva que a 'Tatu-53' (tabela 4) .

Não se notaram diferenças significativas na produção de grãos com a prática de amontoa, sendo que essas diferenças não ocorreram mesmo quando se considerou a interação entre os tratamentos usados (tabela 4) .

De acordo com a figura 1 verificamos que as cultivares 'Tatu-53' e 'Tatu-Lusitânia' sem a utilização de fertilizantes e sem amontoa, produziram um menor número de vagens de amendoim por hectare quan-

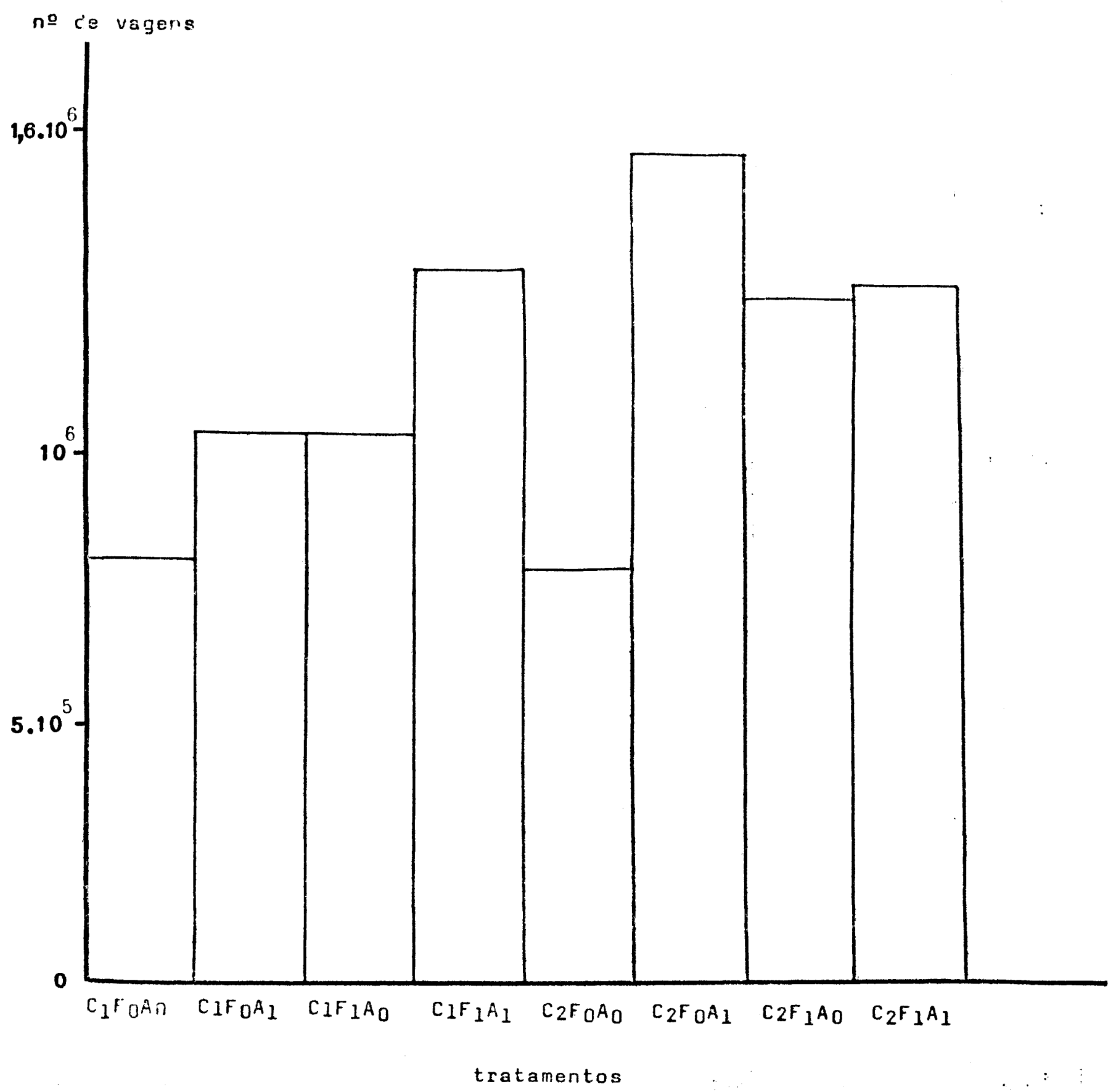

Figura 1 - Número de vagens de amendoim produzidas por hectare nos 8 tratamentos 
do comparadas com as mesmas cultivares submetidas às práticas culturais isolada ou conjuntamente.

A produção de hastes e folhas mostrou-se mais elevada na cultivar 'Tatu-Lusitania' com fertilizantes, seguida da mesma cultivar sem fertilizantes. A cultivar 'Tatu-53' produziu menor peso de matéria seca da palhada, sendo que a ausência de N-P-K reduziu ainda mais este peso (figura 2).

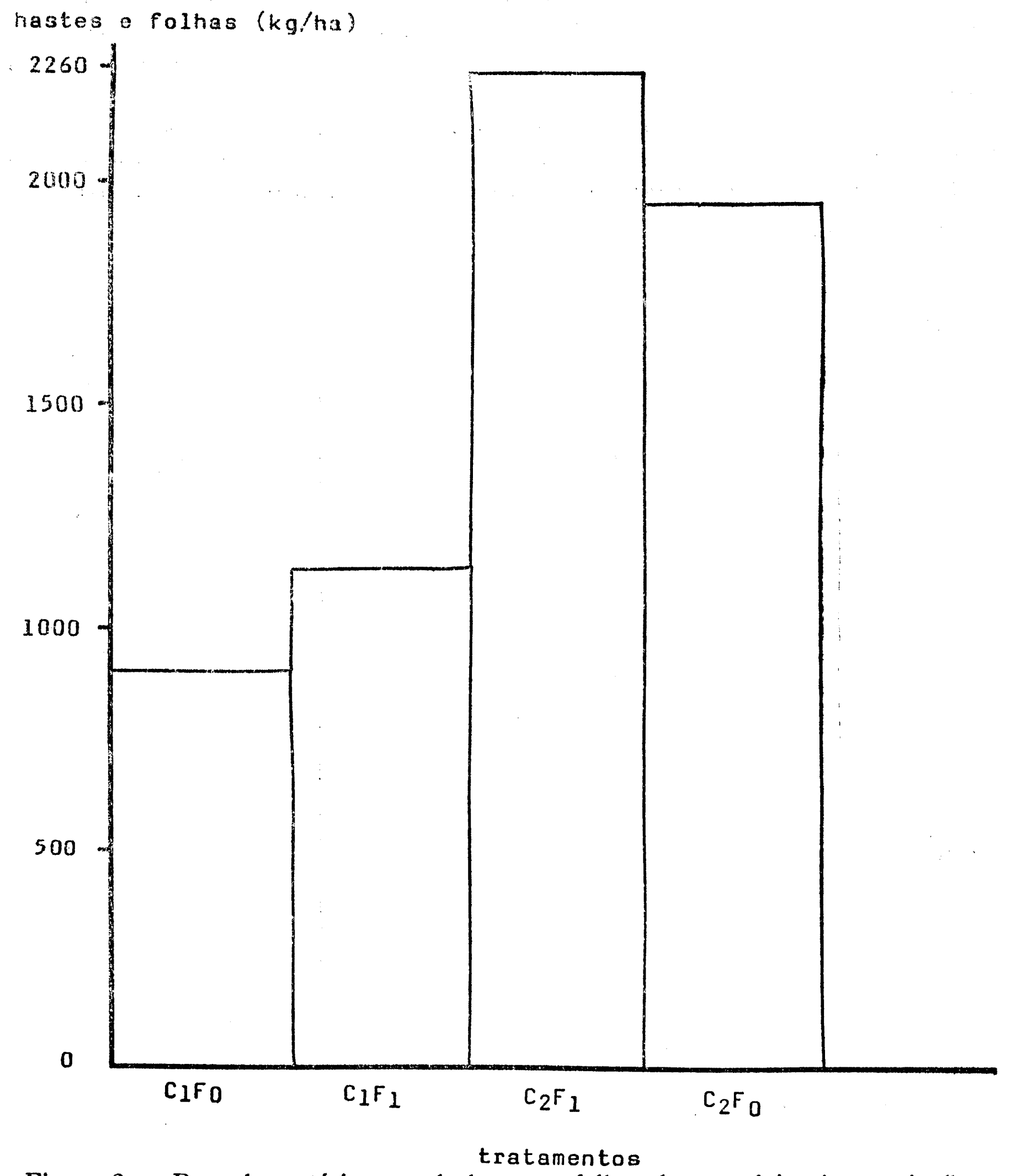

Figura 2 - Peso da matéria seca de hastes e folhas de amendoinzeiro $\mathrm{em} \mathrm{kg} / \mathrm{ha}$, de duas cultivares, com e sem fertilizante. 
Pela figura 3 verificou-se uma diferença marcante no peso da matéria seca das hastes de amendoinzeiro. Notou-se que a cultivar 'Tatu-Lusitânia' mostrou maior peso seco das hastes em relação a 'Tatu-53' . Observou-se ainda que a adubação com N-P-K promoveu um aumento no peso das hastes 70 dias após a semeadura em ambas as cultivares.

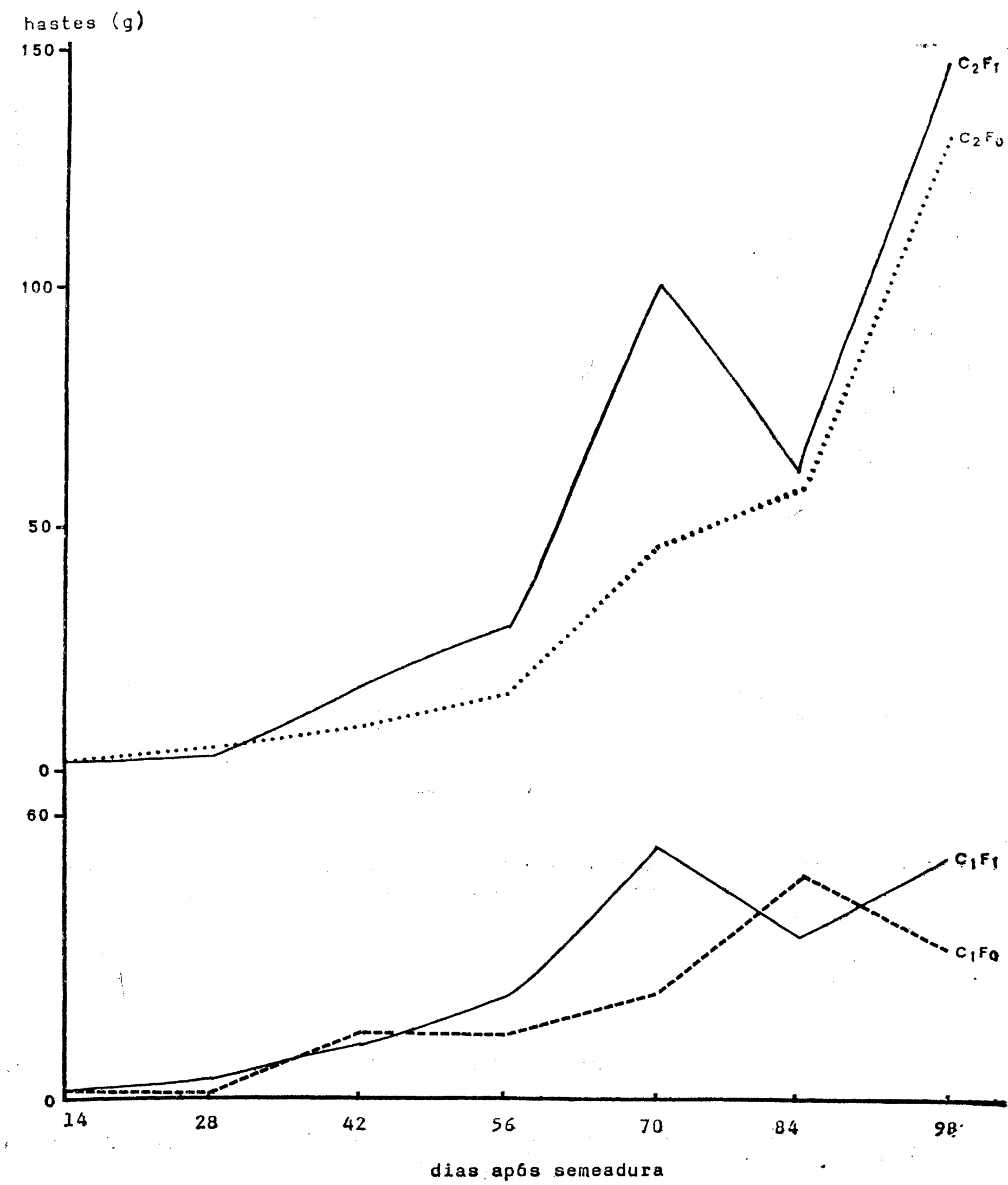

Figura 3 - Peso da matéria seca de hastes de 3 plantas de amendoinzeiro em $g$, de duas cultivares, com e sem fertilizante, no decorrer do ciclo da cultura. 
A utilização de fertilizantes não alterou pronunciadamente o peso da matéria seca foliar do amendoinzeiro. Observou-se que este parâmetro também mostrou valores mais elevados na cultivar 'Tatu-Lusitânia' em relação a 'Tatu-53'. A produtividade foliar mostrou-se mais intensa 70 dias após a semeadura (figura 4).

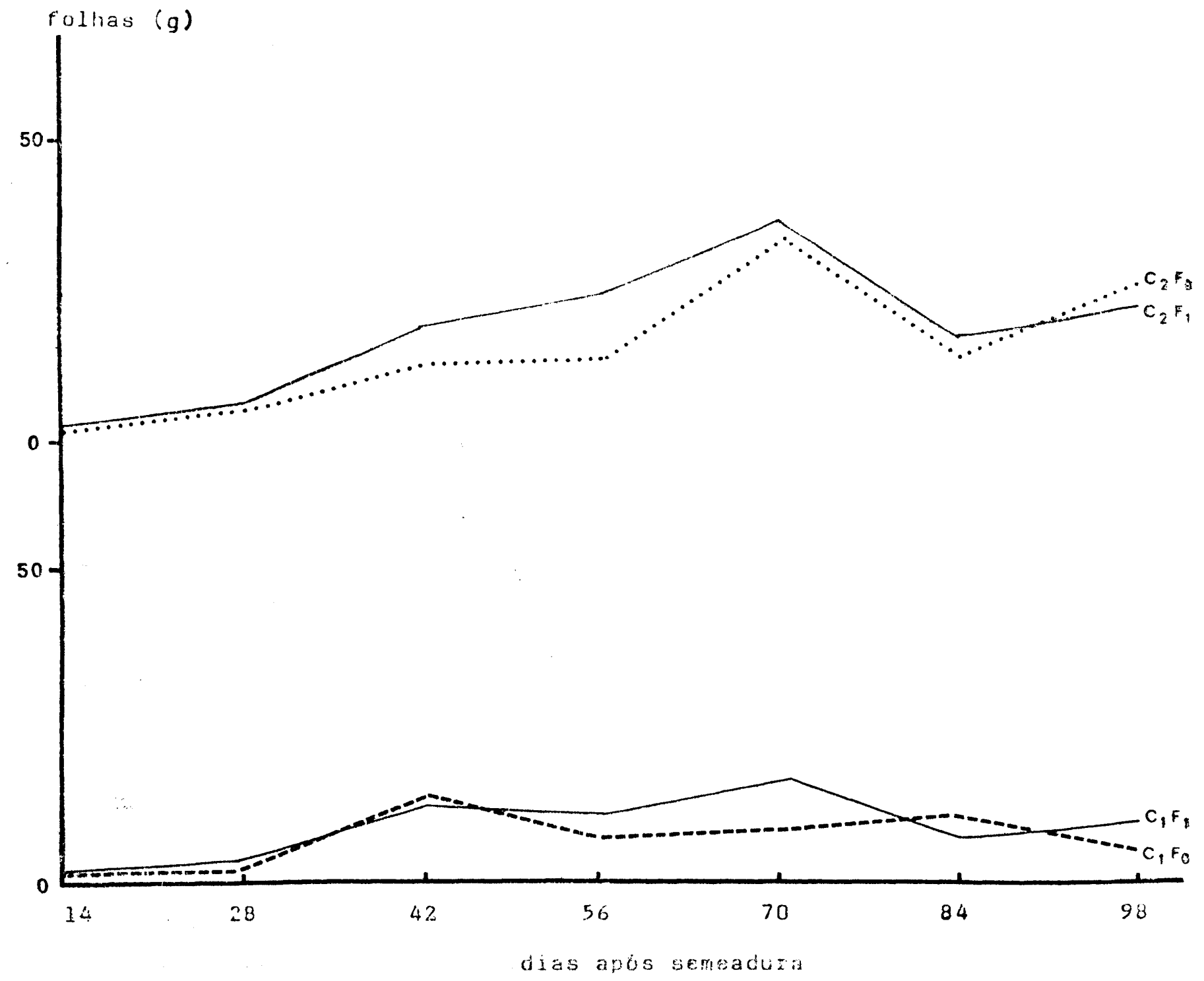

Figura 4 - Peso da matéria seca de folhas de 3 plantas de amendoinzeiro em $\mathrm{g}$, de duas cultivares, com e sem fertilizante, no decorrer do ciclo da cultura

\section{CONCLUSOES}

Dos resultados obtidos neste ensaio, podemos estabelecer as seguintes conclusões:

1. A cultivar 'Tatu-Lusitânia' mostra maior peso das vagens de amendoim em relação a cultivar 'Tatu-53'.

2. O peso dos grãos de amendoim é mais elevado na cultivar 'Tatu-Lusitânia' quando comparada com 'Tatu-53' . 
3. A adubação e a prática da amontoa não afetou o peso das vagens ou o peso dos grãos das cultivares estudadas.

\section{Adubação e amontoa parecem aumentar o número de vagens do amendoinzeiro nas condições ëstudadas.}

5. Ocorre uma tendência de aumento na produção de hastes e folhas com a prática de adubação.

6. A produtividade primária da cultivar 'Tatu-Lusitânia' é superior a produtividade da 'Tatu-53'.

SUMMARY

EFFECTS OF CULTIVATION, FERTILIZING, AND VARIETAL CHARACTERS IN THE PRODUCTIVITY OF PEANUTS (Arachis hypogaea L.)

A trial was carried out in Jaboticabal, São Paulo State, to verify the effect of cultivation, fertilizing, and varietal characters in the productivity of peanut plants.

Heaping and fertilizing 9:30:16 of N-P-K, $250 \mathrm{~kg} / \mathrm{ha}$ were tested comparatively with the check treatments between cultivars "Tatu - 53" and "Tatu - Lusitânia" under field conditions.

The production of peanut shelled or not shelled were not significantly affected when fertilization and cultivation (heaping) were done or when fertilization and cultivation were associated.

There was, yet, a significant difference between the productivity of shelled and not shelled peanuts of the cultivars "Tatu - 53" and "Tatu - Lusitânia".

It was noted however, that the fertilizing practices gaves an increase in the number of peanut shells per hectare when compared with the production of not fertilized or cultivated plants.

There was a great difference between the plant dry weigth of the cultivars "Tatu 53" and "Tatu - Lusitânia". The latter was twice as productive.

Both cultivars developed similarly, though "Tatu - Lusitânia" showed more vigorous stems and leaves.

\section{LITERATURA CITADA}

CANECCHIO F.․, V.; TELLA, R.; ABRAMIDES, E. 1957. Ensaios de variedades de amendoim. II. Oitava e nona série de ensaios. Bragantia 16: 139-145.

CONAGIN, C.H.T.M. 1958. Descrição de algumas variedades do amendoim cultivado (Arachis hypogaea L.). Bragantia 17: 311-330.

GARGANTINI, H.; TELLA, R.; CONAGIN, A. 1958. Ensaio de adubação N-P-K em amendoim. Bragantia 17: 1-12.

GRANER, E.A.; GODOY JR. C. 1967. Culturas da fazenda brasileira. Ed. Melhoramentos, Sã́o Paulo, $461 \mathrm{p}$.

GREENWOOD, M. 1951. Fertilizer trials with groundnuts in Northern Nigeria. Emp. J. Exp. Agr. 19:225-241.

MALAVOLTA, E. 1963. Nutrição mineral da planta do amendoim. Supl. Agr. Est. São Paulo 422: 10.

NAKAGAWA, J.; NAKAGAWA, J.; BOARETTO, A.E. 1973. Ensaio com doses crescentes de nitrogênio, fósforo e potássio na cultura do amendoim (Arachis hypogaea L.). Rev. Agric. 48: 37-44.

TELIA, R.; CANECCHIO F., V.; ROCHA, J.L.V.; FREIRE, E.S.; IGUE, T. 1970. Efeito de doses crescentes de nitrogênio, fósforo e potássio sobre a produção de amendoim em solos podzolizados de Lins e Marilia. Bragantia 29: 199-205.

TELLA, R.; CANECCHIO F., V.; ROCHA, J.L.V.; FREIRE, E.S.; IGUE, T. 1971. Efeito da adubação do amendoim com nitrogênio, fósforo e potássio, na ausência e na presença de calcário. Bragantia 30:39-47. 
